\title{
Minimal-invasive Gefäßeingriffe unter Röntgenkontrolle: Radiologische Interventionen gehören in die Radiologie!
}

\author{
Stellungnahme der Fachgesellschaften und Berufsverbände der Radiologie und der Neuro- \\ radiologie zur aktuellen Diskussion der MWBO
}

Die Interventionelle Radiologie, das heißt die bildgestützte minimal-invasive Gefäßtherapie mittels Kathetertechniken, genießt in Deutschland einen hervorragenden Ruf. Viele alters- und lebensstil-bedingte Erkrankungen der Blutgefäße wie etwa die periphere arterielle Verschlusskrankheit (pAVK) lassen sich auf diese Weise wirkungsvoll, patientenschonend und kostengünstig behandeln. Seit über 60 Jahren werden die Kompetenzen auf dem Gebiet der minimal-invasiven Medizin im Fach Radiologie gepflegt und ständig weiterentwickelt. Eine Reklamation dieser Therapieverfahren durch andere medizinische Fachgebiete, wie sie in der aktuellen Diskussion um die Musterweiterbildungsordnung (MWBO) der Bundesärztekammer erfolgt, schadet der Versorgungsqualität, führt zu einer vermehrten Strahlenbelastung der Patientinnen und Patienten und treibt durch unkontrollierbare Leistungsausweitungen die Kosten im Gesundheitswesen in die Höhe. Die Fachgesellschaften und Berufsverbände der Radiologie und der Neuroradiologie fordern deshalb die Nachbarfächer und die Bundesärztekammer auf, beim Prinzip der Interdisziplinarität zu bleiben und die bildgestützten interventionell-radiologischen Therapieverfahren in der Radiologie zu belassen.

\section{Radiologen sind klinisch und technisch kompetente Gefäßmediziner!}

Radiologisch gesteuerte Gefäßinterventionen aller Körperabschnitte - außer am Herzen - sind seit über 60 Jahren integraler Bestandteil der Radiologie und haben sich aus der diagnostisch-radiologischen Gefäßuntersuchung mit Kathetern (Angiografie) entwickelt. Die Radiologie war und ist Motor dieser innovativen, patientenscho- nenden Therapieverfahren. Interventionelle Radiologen sind klinisch denkende und handelnde Gefäßmediziner, die die Krankheitsbilder in allen relevanten Facetten kennen, ihre Kompetenz in bildgebenden Verfahren diagnostisch nutzen und die minimal-invasiven interventionellen Techniken beherrschen. Heute setzen interventionelle Radiologen ihre Expertise in allen Feldern der bildgestützten vaskulären Eingriffe ein. Das Spektrum reicht von rekanalisierenden und rekonstruierenden Gefäßinterventionen über gefäßverschließende Maßnahmen, die Etablierung intrahepatischer Shunts bis zu hochkomplexen interventionell-onkologischen Eingriffen. Dieses über Jahrzehnte gewachsene Therapiespektrum belegt die Kompetenz der Radiologen, die in Art und Umfang in keiner anderen Disziplin besteht und die auch in Zukunft eine effektive, effiziente und qualitätsgesicherte Durchführung zum Nutzen der Patienten gewährleistet.

\section{Bildgebung bei Interven- tionen ist Expertensache!}

Minimal-invasive Gefäßeingriffe erfordern die bestmögliche Bildgebung zur Planung und Kontrolle von Interventionen. Die vollständige Kompetenz in Anwendung und Beurteilung aller für die Gefäßmedizin genutzten bildgebenden Verfahren (DSA, Sonografie, CTA und MRA) ist seit Jahrzehnten Kernaufgabe und Kernkompetenz der Radiologie. Der Versuch anderer Fachgebiete, eigene Fachgrenzen auf interventionellradiologische Bereiche auszuweiten, geht Hand in Hand mit Bestrebungen, auch zunehmend Zugriff auf die radiologische Bildgebung zu erhalten.

\section{Die Interventionelle Radio- logie verfügt über das um- fangreichste und beste Qualifizierungs- und Zerti- fizierungssystem!}

Interventionelle Gefäßeingriffe sind seit vielen Jahren fester, in der WBO verankerter Bestandteil der Weiterbildung in Radiologie. Dies hat seit etwa zwei Ärztegenerationen dazu geführt, dass die interventionellradiologische Kernkompetenz eine sehr breite Basis besitzt. Dies ist in keinem anderen medizinischen Fach der Fall. Auf dieser Basis wurden in der Radiologie flächendeckend zusätzliche Spezialkompetenzen entwickelt, weitergegeben und klinisch eingesetzt. Unter dem gemeinsamen Dach ihrer Fachgesellschaften hat die Radiologie in Deutschland ein umfangreiches, differenziertes und inhaltlich äußerst anspruchsvolles Qualifizierungs- und Zertifizierungskonzept für alle minimal-invasiven Interventionen etabliert. Auf Basis ihrer in der aktuellen Weiterbildungsordnung festgeschriebenen, praktisch zu erwerbenden interventionellen Kompetenzen haben bereits über 1.000 Radiologinnen und Radiologen eine mehrstufige zusätzliche Qualifizierung in bildgestützten Interventionen durchlaufen und mit einer umfassenden und objektivierten Fachprüfung nach internationalen Bildungsstandards abgeschlossen. Die interventionell-radiologischen Ausbildungsstätten unterziehen sich zudem einem freiwilligen Zertifizierungs- und Re-Zertifizierungsverfahren. Keine andere Fachgesellschaft, die sich nun interventionelle Verfahren in die Weiterbildungsordnung schreiben lassen möchte, kann auf ein solch komplexes und flächendeckendes Qualifizierungsprogramm mit unabhängig geprüften Qualifizierungsanforderungen zurückgreifen. 


\section{Fortlaufende Qualitätskon-} trolle ist ein fester Bestandteil radiologischer Arbeit!

Voraussetzung für die kontinuierliche Verbesserung der Ergebnisse invasiver Eingriffe ist eine professionelle Qualitätssicherung. Die Radiologie verfügt über ein seit mehr als 25 Jahren kontinuierlich weiterentwickeltes Qualitätssicherungsprogramm, das das gesamte Spektrum der minimal-invasiven Eingriffe unter Bildkontrolle umfasst und an dem inzwischen über 300 Kliniken bundesweit teilnehmen. Alleine im Jahr 2016 wurden in der Datenbank der Deutschen Gesellschaft für Interventionelle Radiologie und minimal-invasive Therapie (DeGIR) über 170000 radiologische Interventionen hinsichtlich Prozess- und Ergebnisqualität transparent dokumentiert. Die Dokumentation reicht von der klinischen Indikation über relevante Eckdaten der einzelnen Eingriffe bis zur individuellen Strahlenbelastung durch den Eingriff sowie das klinische Ergebnis und die Komplikationsrate. Keine andere medizinische Disziplin, die jetzt im Rahmen der Novellierung der MWBO die sogenannten „endovaskulären Eingriffe“ bzw. „endovaskulären Operationen" für sich beansprucht, hat ein solches Programm auch nur annähernd etabliert.

\section{Gefäßpatienten sind auf interdisziplinäres Arbeiten}

\section{angewiesen!}

Interventionelle Radiologen sind aufgrund der Ausrichtung ihres Faches interdisziplinär denkende und arbeitende Ärzte. Die Radiologie ist in vielen Bereichen der klinischen Medizin, gerade aber auch in der Gefäßmedizin, das Querschnittsfach in der stationären und ambulanten Versorgung. Im klinischen Alltag stimmen sich interventionelle Radiologen eng mit den anderen gefäßmedizinischen Fächern (operative Gefäßchirurgie, internistische Angiologie, vaskuläre Neurologie, Diabetologie und Neph- rologie) ab und handeln ganz überwiegend auf Zuweisung. Gefäßpatienten benötigen genau diese kompetente, interdisziplinäre Versorgung, in der alle Fächer ihre Kernkompetenzen einbringen. Dies gilt insbesondere auch für den Betrieb sogenannter Hybrid-OPs, die in den letzten Jahren in gefäßchirurgischen Einheiten zu oft unkritisch installiert und nicht ausreichend wirtschaftlich betrieben wurden. Hybrid-Eingriffe können nur durch gemeinsames Handeln von Radiologen und Gefäßchirurgen erfolgreich sein, sodass dem Patienten die beste chirurgisch-operative und die beste interventionell-radiologische Kompetenz zugutekommt. Dies ist bislang in vielen Kliniken auch gelebte und funktionierende Praxis.

\section{Nur die Radiologie ermög- licht eine flächendeckende}

\section{4/7-Versorgung!}

Im Gegensatz zu den Fächern, die sich jetzt um die Weiterbildungsbefugnis für radiologische Interventionen bemühen, stellt die Radiologie bereits heute, aufgrund ihrer umfassenden Expertise, eine Flächen deckende, zeitlich uneingeschränkte und höchsten Qualitätsanforderungen genügende, ambulante wie stationäre Versorgung mit allen bildgestützten vaskulären Eingriffen sicher. Radiologen sind viel besser als Ärzte anderer Disziplinen mit allen Materialien und Methoden der minimal-invasiven vaskulären Verfahren vertraut. Wie erfolgreich und rasch auch neue $\mathrm{He}$ rausforderungen in ihrer gesamten Breite angenommen und in der klinischen Praxis etabliert werden, wird am Beispiel der endovaskulären Therapie des Schlaganfalls, die erst 2015 wissenschaftliche Evidenz erlangte, eindrucksvoll belegt. Über 170 radiologische und neuroradiologische Zentren gewährleisten deutschlandweit eine 24/7-Betreuung bei der minimal-invasiven Akutbehandlung des ischämischen Schlaganfalls. Würden durch die neue MWBO radiologische Interventionen zunehmend anderen Fächern zugeteilt, würden eine qualitätsgefährdende Diversifizierung von Kompetenzen und eine erhebliche Kostensteigerung resultieren.

\section{Alles andere als trivial: der Strahlenschutz!}

Interventionelle Gefäßeingriffe gehören laut Bundesamt für Strahlenschutz (BfS) zu den Maßnahmen in der Medizin mit der höchsten Strahlenexposition für den Patienten. Radiologen sind die mit Abstand am besten im Strahlenschutz aus- und weitergebildeten Ärztinnen und Ärzte. Gemäß Röntgenverordnung ist in der Radiologie eine mindestens dreijährige, umfangreiche Weiterbildung im Strahlenschutz verankert. Hierdurch wird bei Radiologen ein breites Verständnis für Angelegenheiten des Strahlenschutzes etabliert, das auch nach der Weiterbildung im klinischen Alltag rund um die Uhr fester Bestandteil radiologischen Handelns ist. Die normativen Vorgaben für den Strahlenschutz wurden in den letzten Jahren fortwährend verschärft, dies wird sich ab 2018 durch neue EU-Vorgaben weiter verstärken. Die neue MWBO muss dieser Tatsache Rechnung tragen, indem sie interventionell-radiologische Eingriffe auch weiterhin der Radiologie zuordnet. Eine Ausweitung der Methoden auf nichtradiologische Fächer, die nur über eine nebenbei absolvierte Schmalspurausbildung im Strahlenschutz verfügen, konterkariert die eminent wichtige Bestrebung, die medizinische Strahlenexposition für Patientinnen und Patienten zu senken.

\section{Radiologische Interventio- nen in der Radiologie: Auch ein Gebot der Ökonomie!}

Die für Gefäßinterventionen notwendigen bildgebenden Systeme erfordern hohe Investitionen, die ökonomischen Zwänge im Gesundheitswesen hingegen eine immer effizientere Ressourcennutzung. Es ist daher auch ökonomisch unsinnig, interventio-

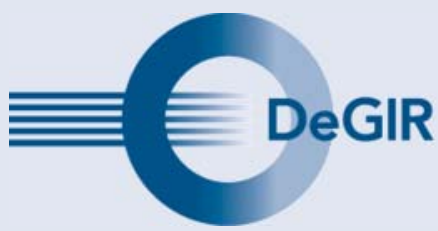

\section{DEUTSCHE GESELLSCHAFT FÜR INTERVENTIONELLE RADIOLOGIE}

UND MINIMAL-INVASIVE THERAPIE

in der Deutschen Röntgengesellschaft e.V. 
nell-radiologische Verfahren auf andere Fächer auszuweiten, zumal in den Radiologien der Weiterbildungsstätten sämtliche Ressourcen (z. B. Angiografiesysteme, Fachpersonal) schon heute vorhanden sind. Die Erfahrung zeigt, dass die derzeit von bestimmten Fächern gewünschten Änderungen in der MWBO auch zu Forderungen nach eigenen Geräten, Personal und Räumlichkeiten führen wird. Zudem hätte die MWBO-Festschreibung neuer Leistungsbereiche zweifellos Indikationsausweitungen zwecks Fallzahlgenerierung zur Folge, um die Vorgaben der Weiterbildungsordnung auch erfüllen zu können. Hinzu kommen ein interner Wettbewerbsdruck in Wei- terbildungsstätten und persönliche $A b$ rechnungsanreize durch niederschwellige Selbstzuweisung bei gleichzeitigem Wegfall des Mehraugenprinzips, die die unkontrollierte Leistungsausweitung befördern. All dies kann gesundheitspolitisch nicht gewollt sein.

\section{Was sich in der Vergangen- heit bewährt hat, sollte auch für die Zukunft Bestand}

\section{haben!}

Die Deutsche Gesellschaft für Interventionelle Radiologie und minimal-invasive The- rapie (DeGIR), die Deutsche Röntgengesellschaft (DRG), die Deutsche Gesellschaft für Neuroradiologie (DGNR), der Berufsverband der Radiologen (BDR) und der Berufsverband der Neuroradiologen (BDNR) fordern, dass die bisherigen Fachgrenzen bezüglich interventionell-radiologischer Eingriffe nicht verschoben werden. Nur so ist gewährleistet, dass derartige Eingriffe auch weiterhin klinisch-rational, mit gesicherter Qualität, ressourcenschonend und gesamtökonomisch sinnvoll durchgeführt werden. 